\title{
Feasibility of a de-epithelialized orbicularis oculi musculocutaneous flap for subbrow blepharoplasty
}

\author{
Seong Yeon Kim, Ma Rhip Ahn, \\ Young Chul Suh, Young Jin Kim, \\ Jung Ho Lee
}

Department of Plastic and Reconstructive Surgery, College of Medicine, The Catholic University of Korea, Seoul, Korea

\begin{abstract}
Background Subbrow blepharoplasty (SBB) has advantages over traditional blepharoplasty in that it results in more natural-looking creases and contours and is more effective for lateral periorbital rejuvenation. However, in older patients with sunken upper eyelid, this procedure might make the superior eyelid sulcus appear worse because the thicker upper eyelid tissue in the subbrow area is replaced by thinner tissue. To overcome this limitation, we developed a de-epithelialized musculocutaneous flap for SBB. Methods From August 2016 to January 2018, 13 patients with sunken upper eyelid deformity underwent SBB. For each of these patients, a typical SBB incision was made, but the tissue usually removed in SBB was dissected as a flap based on the branch of the ophthalmic vessel. After elevation, the flap was transposed or folded to correct the sunken deformity. The results were assessed by the patients themselves and by three plastic surgeons at 6 months postoperatively.

Results The mean age of the patients was 63 years (range, $47-81$ years). There were no postoperative complications related to wound dehiscence, hypertrophic scarring, or sensory changes. All patients and plastic surgeons provided scores indicating good to excellent results and were satisfied with the cosmetic outcomes. All patients reported improved functional visual acuity after surgery.

Conclusions Our technique is simple and effectively addresses both sunken upper eyelid deformity and dermatochalasis. Unlike fat or dermofat grafting, our new technique does not require an additional donor site, and it is more reliable in terms of vascularity.
\end{abstract}

Keywords Blepharoplasty / Eyelids / Myocutaneous flap

\section{INTRODUCTION}

Subbrow blepharoplasty (SBB) was designed to overcome the disadvantages of classic upper eyelid blepharoplasty by excising extra skin, subcutaneous adipose tissue, and the orbicularis oculi muscle

Received: May 16, 2019 Revised: Jun 19, 2019 Accepted: Jun 20, 2019 Correspondence: Jung Ho Lee Department of Plastic and Reconstructive Surgery, Bucheon St. Mary's Hospital, College of Medicine, The Catholic University of Korea, 327 Sosa-ro, Wonmi-gu, Bucheon 14647, Korea Tel: +82-32-340-7059, Fax: +82-32-340-2666, E-mail: tfm0822@catholic. ac.kr

Copyright @ 2019 The Korean Society for Aesthetic Plastic Surgery.

This is an Open Access article distributed under the terms of the Creative Commons Attribution Non-Commercial License (http://creativecommons.org/licenses/by-nc/4.0/) which permits unrestricted non-commercial use, distribution, and reproduction in any medium, provided the original work is properly cited. www.e-aaps.org along the lower border of the eyebrow. Compared with classic blepharoplasty, SBB is more effective for the correction of lateral eyelid skin drooping and results in a more natural-looking eyelid crease [1].

However, SBB has certain limitations. The upper eyelid skin is thickest just below the eyebrow [2], which is excised in SBB. Consequently, following the resection of redundant tissue, the thick skin below the eyebrow needs to be sutured to the thinner eyelid skin, which can cause stair-step deformities. In addition, the supraorbital area, which is covered by thick skin and subcutaneous tissue before surgery, is covered postoperatively by thinner tissue, which may make the sunken upper eyelid more visible if the patient has tissue atrophy between the orbital rim and upper border of the globe.

In this study, we tried to achieve successful periorbital rejuvenation and sunken upper eyelid correction in patients with sunken 
upper eyelids and dermatochalasis using a modified form of SBB. In this modified technique, instead of excision, the skin and subcutaneous tissue in the subbrow area are elevated as a flap, which is then used to correct the sunken deformity.

\section{METHODS}

\section{Anatomical basis of the flap}

Our newly developed method is relatively simple and safe, but requires knowledge of the blood supply to the orbicularis oculi muscle, which originates from three main sources [3]. The first blood source is the orbitozygomatic branch originating from the superficial temporal artery, which travels into the muscle at a point $2 \mathrm{~cm}$ from the lateral side of the muscle. The second source is the branch of the angular artery, which runs into the muscle at a point $1 \mathrm{~cm}$ from the medial side of the muscle. The third source is the ophthalmic artery (Fig. 1A). Therefore, a versatile orbicularis oculi musculocutaneous flap can be created and utilized if the branch at the medial or lateral portion can be preserved. There are many publications describing the application of anatomical knowledge to develop techniques that use an orbicularis oculi musculocutaneousbased island flap to cover upper eyelid defects following trauma or tumor removal [4-7]. Using a combination of anatomical knowledge and data from previously published clinical studies, we developed a technique to create a de-epithelialized orbicularis oculi mus-

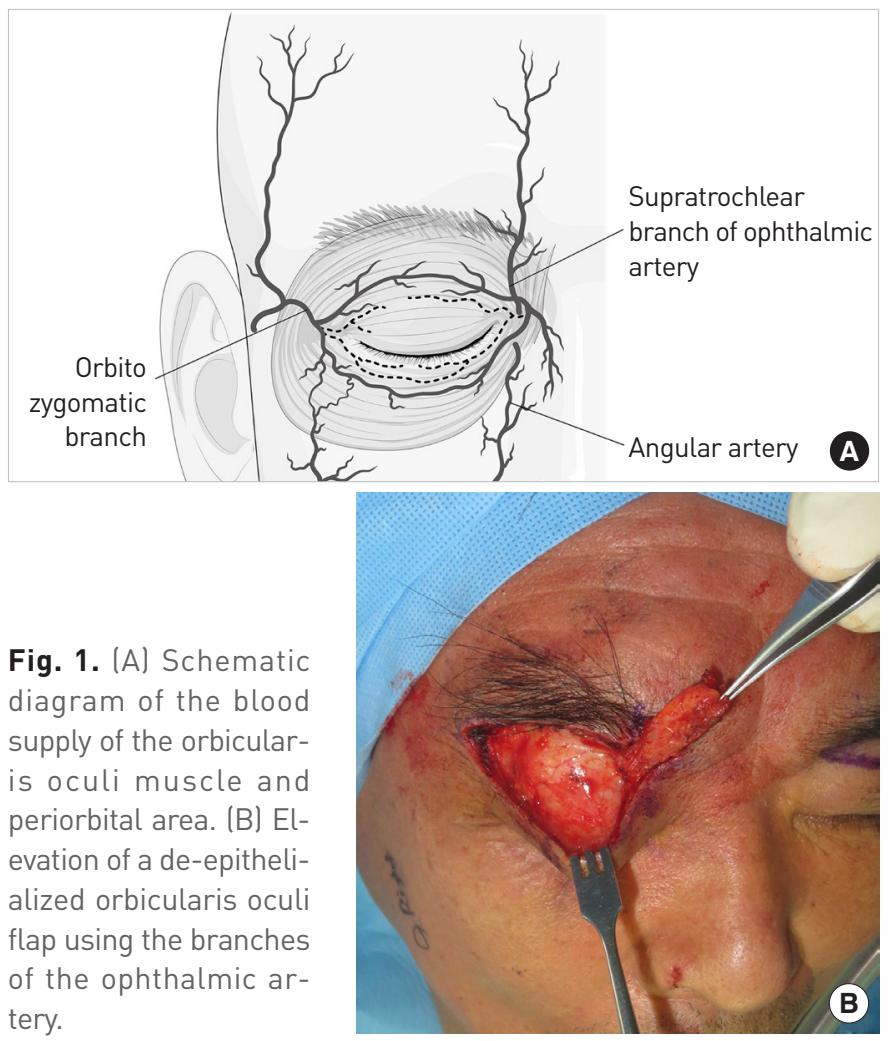

culocutaneous flap, with a branch of the ophthalmic artery, to correct sunken upper eyelid deformities (Fig. 1B).

In our clinic, we recommend SBB for patients who have dermatochalasis and want a natural look without a double fold. In such patients, we performed modified SBB for the simultaneous correction of dermatochalasis and sunken upper eyelid in patients who presented with sunken upper eyelid. Sunken upper eyelid was identified in the primary position of the gaze and after cephalic traction of the redundant upper eyelid. Additionally, patients with sunken upper eyelid and mild-to-moderate ptosis underwent modified SBB if they refused a double-fold operation or natural rejuvenation.

From August 2016 to January 2018, 13 patients were recruited for the study (11 female and 2 male). The mean age of the patients was 63 years (range, $47-81$ years) and each patient was followed up for 6 months postoperatively (Table 1). The postoperative results were scored as follows: dissatisfied, 0 ; fair, 1; good, 2; or excellent, 3 . Six months postoperatively, each patient was given a questionnaire to complete; overall satisfaction was scored in terms of the aesthetic outcome and scar evaluation. In addition, three plastic surgeons (JWS, HWS, and YJL) who were blinded to the specific details of each case also evaluated each patient's clinical photographs and rated their overall satisfaction in terms of the aesthetic outcome and scar evaluation.

The study was approved by the Institutional Review Board of Bucheon St. Mary's Hospital (IRB No. HC18REDI0094) and was performed in accordance with the principles of the Declaration of Helsinki.

\section{Surgical technique}

Surgical planning was performed with the patient in a sitting position. To prevent pedicle injury, the supraorbital foramen was first palpated and then marked. In addition, we also marked the sunken area in the upper eyelid. Next, the superior incision line was designed along the inferior border of the eyebrow, and the amount of skin to be excised was determined by the pinch test. The maximum possible amount of redundant skin at the lateral side of the eyelid was excised.

Under local anesthesia, the skin was excised, and de-epithelialization was performed. The musculocutaneous flap was then elevated from the lateral to medial direction. In a position close to the

Table 1. Patient characteristics and follow-up period

\begin{tabular}{lc}
\hline Characteristic & Value $(\mathrm{n}=13)$ \\
\hline Sex & \\
Male & 2 \\
Female & 11 \\
Age, mean (range), yr & $63(47-81)$ \\
Follow-up period, mo & 6
\end{tabular}



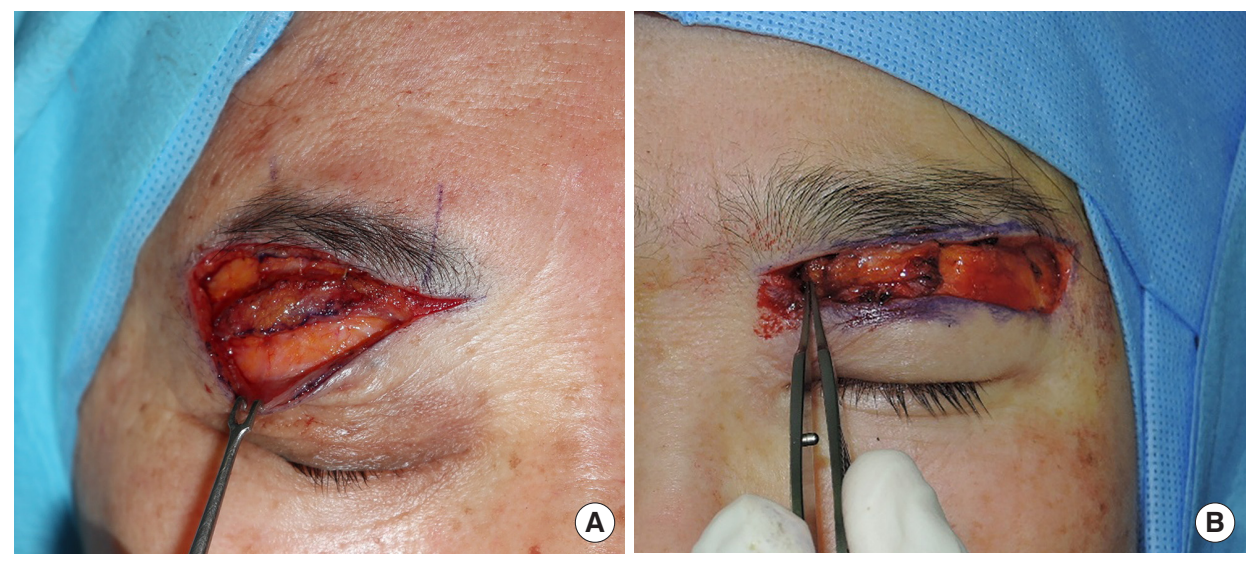

Fig. 2. (A) Orbicularis oculi musculocutaneous flap transposed over a sunken area. (B) Folding of a thin orbicularis oculi musculocutaneous flap to fill the sunken area.

supraorbital foramen, we stopped the submuscular dissection and performed subcutaneous undermining in order to prevent pedicle injury. After elevation, and to inset the flap snugly, a submuscular pocket between the orbital septum and orbicularis oculi was created over the sunken area. According to the amount of sunken deformity, any flap redundancy was excised. If the flap thickness was sufficient, the flap was medially transposed over the sunken area. If the flap was thin, however, it needed to be folded (Fig. 2). Then, the elevated flap was fixed to the periosteum of the supraorbital rim using 5-0 Vicryl sutures, and the surgeon ensured that the sunken deformity and any other irregularities were corrected. After flap inset, the orbicularis oculi muscle along the inferior incision margin was fixed to the periosteum of the supraorbital rim using 5-0 Vicryl sutures to prevent drooping of the eyelid skin. The skin was temporarily closed to check for flap-induced bulkiness, and an inset flap was thinned if bulkiness was observed. Then, the subcutaneous tissue was sutured using 5-0 Vicryl sutures and the skin was closed using 6-0 silk sutures. The deep dermis of the lower flap was approximated with the superficial dermis of the upper flap to prevent a stair-step deformity secondary to the difference in skin thickness. On postoperative day 1 , the surgeon checked for complications, such as hematoma, wound disruption, or asymmetry. All sutures were removed on postoperative day 5 .

\section{RESULTS}

During the 6-month follow-up period, there were no complications, such as hematoma, wound dehiscence, or infection. The mean size of the flaps was $10.7 \mathrm{~mm}$ (width) $\times 46 \mathrm{~mm}$ (length). Overall aesthetic satisfaction, on a scale of 0 to 3 , was rated as 2.85 by the patients and 2.59 by the plastic surgeons, indicating good to excellent results. The evaluation of visible scars also yielded good to excellent results ( 2.23 and 2.44 by the patients and the plastic surgeons, respectively) (Table 2).
Table 2. Postoperative mean satisfaction as judged by patients and plastic surgeons

\begin{tabular}{lcc}
\hline Result & Patients & Plastic surgeons \\
\hline Aesthetic satisfaction & $2.85 \pm 0.38$ & $2.59 \pm 0.50$ \\
Scar & $2.23 \pm 0.60$ & $2.44 \pm 0.55$ \\
Complications & None & - \\
\hline
\end{tabular}

Values are presented as mean \pm SD.

Scores: 0 , dissatisfied; 1 , fair; 2 , good; 3 , excellent.

\section{Case 1}

A 65-year-old man visited our clinic with a drooping eyelid, visual interference, and skin eruption in the lateral canthal area due to maceration caused by contact of the upper and lower eyelids (Fig. 3A). Upon physical examination, we noted severe upper eyelid hooding. In addition, a sunken deformity was observed with cephalic traction of the eyebrow (Fig. 3B). To correct the upper eyelid drooping and sunken upper eyelid, we planned to apply the modified SBB technique. The skin incision was designed as described above. Following skin incision and de-epithelialization, a musculocutaneous flap was elevated and an inferior submuscular pocket was created to inset the flap. Then, the flap was transposed over the sunken area and the superior margin of the flap was fixed to the periosteum of the supraorbital rim. At the 6-month follow-up, the patient's eyelid hooding had visibly improved, and the sunken deformity had been corrected (Fig. 3C and D).

\section{Case 2}

A 56-year-old woman visited our clinic with upper eyelid skin drooping and a reduced visual field. Upon physical examination, we observed upper eyelid laxity and a sunken deformity (Fig. 4A). To correct the eyelid laxity and sunken deformity simultaneously, we performed our modified SBB technique. At the 6-month followup, periorbital rejuvenation was observed, and the patient's sunken deformity had improved (Fig. 4B). 

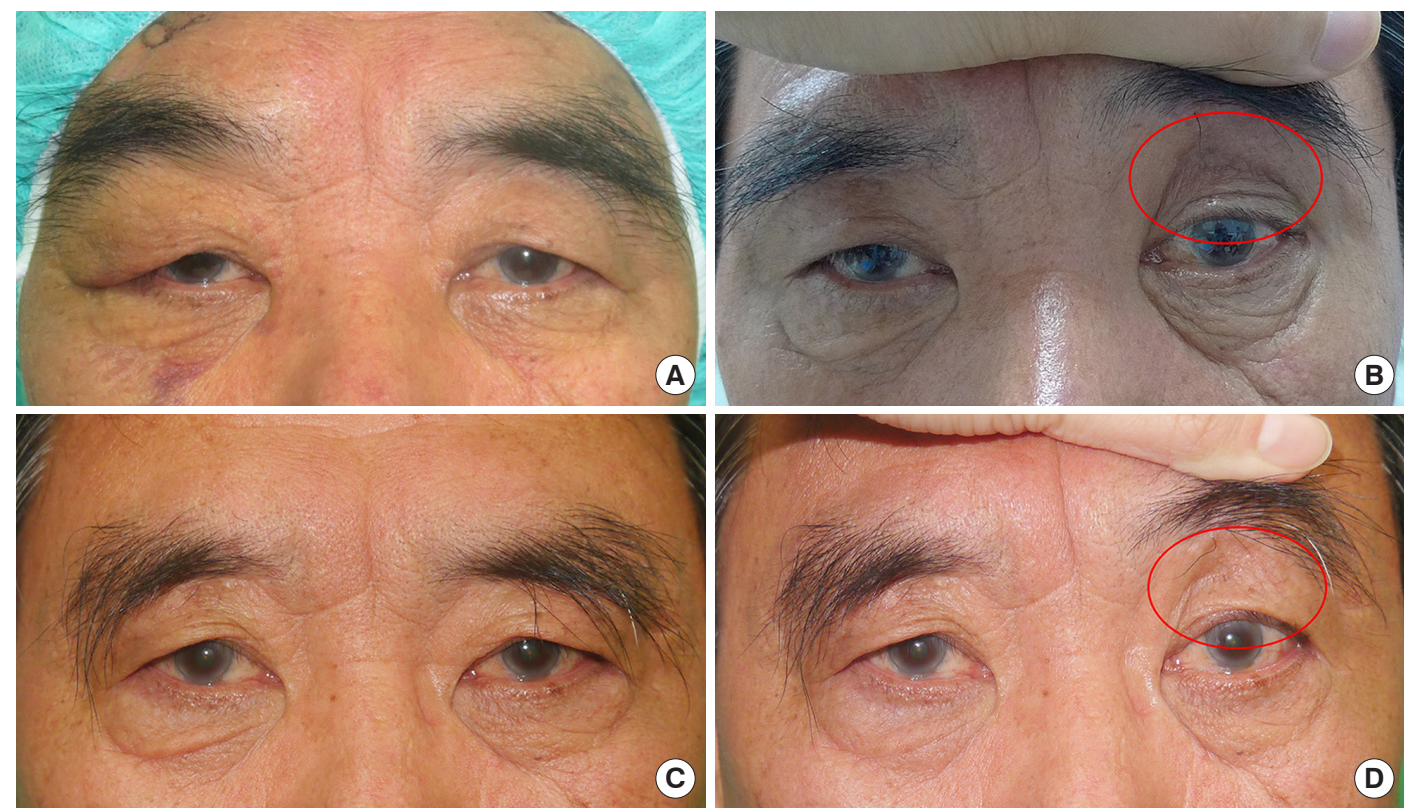

Fig. 3. Case 1. (A) A 65-year-old man with dermatochalasis. (B) With cephalic traction of the upper eyelid skin, sunken upper eyelid was noted (circle). (C) Six months postoperatively. (D) Six months postoperatively, with cephalic traction of the upper eyelid skin. The sunken upper eyelid was successfully corrected after surgery (circle).
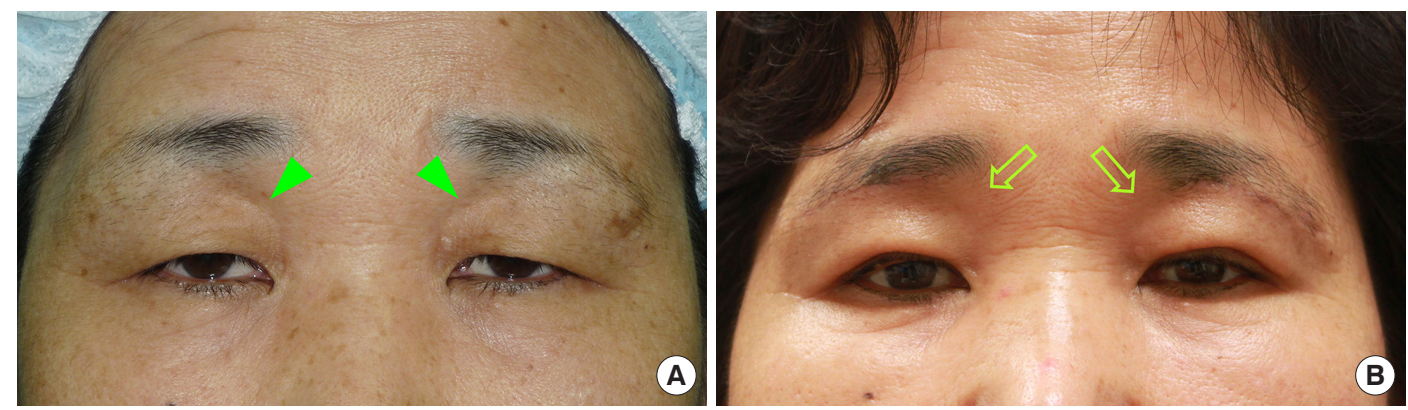

Fig. 4. Case 2. (A) A 56-year-old woman with lateral upper eyelid hooding and sunken upper eyelids (green arrow heads). (B) Six months postoperatively. The sunken upper eyelid was successfully corrected after surgery (green blank arrows).

\section{DISCUSSION}

Brow ptosis, blepharoptosis, dermatochalasis, and loss of periorbital volume can all occur with increasing age. In some older patients, the fold of the upper eyelid skin droops and creates a hood, thereby interfering with vision [8]. If the accumulation of excess skin in the area below the lateral third of the eyebrow is particularly prominent, then contraction of the frontalis muscle against gravity gradually weakens with age, resulting in lowering of the lateral third of the eyebrow [9].

Because of the typical anatomical characteristics of Asian patients, such as thicker eyelid skin and a higher position of the eyebrows, lateral hooding can be more significant in older Asian patients. Therefore, in such patients, traditional blepharoplasty is often inadequate for the removal of redundant upper eyelid skin and can result in scarring beyond the lateral canthal area, giving rise to unnatural postoperative results [10].

SBB was developed to overcome the limitations of traditional blepharoplasty [11]. By removing redundant skin and subcutaneous tissue in the subbrow area, this technique leaves no visible scar and creates a natural supratarsal crease. Another advantage is that compared with traditional blepharoplasty, more upper eyelid skin in the lateral area can be excised, with minimal dog-ear scarring [12]. However, because the skin and subcutaneous tissue in the subbrow area are much thicker than the tissue in the supraciliary area, there is the potential to leave an unnatural infra-brow scar, such as a stair-step deformity [2]. In addition, if the patient has a supraorbital sunken eyelid deformity, then it may become more visible postoperatively because redundant tissue over the sunken area is replaced by thinner tissue. 

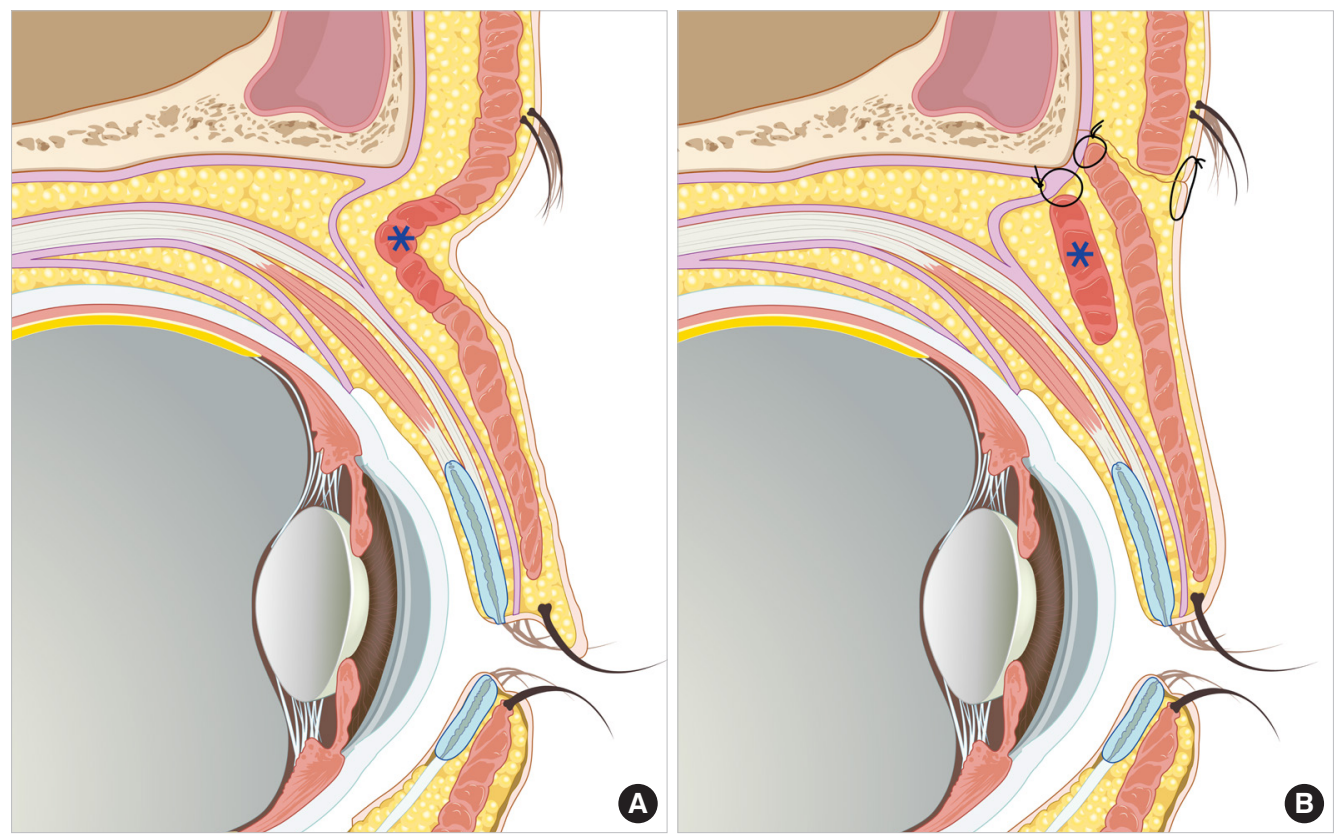

Fig. 5. (A) Preoperative sagittal section of a sunken upper eyelid in an elderly individual showing atrophy of the orbital fat. The asterisk represents the musculocutaneous flap that would be used to correct the sunken upper eyelid. (B) Postoperative sagittal section after modified subbrow blepharoplasty showing how a de-epithelialized musculocutaneous flap can be used to reinforce orbital fat atrophy and how the orbicularis oculi muscle in the lower flap can be fixed to the periosteum. This technique allows us to correct sunken upper eyelid deformities, yielding a highly satisfactory contour.

A sunken deformity is defined as a depression between the eyebrow and the upper border of the globe [13]. With aging, sunken upper eyelids can develop due to skin laxity and atrophy of orbital fat and subcutaneous tissue. In addition, with the increasing number of blepharoplasty procedures in the Asian population, sunken upper eyelids are becoming increasingly common because the current technique for blepharoplasty in Asian patients involves removing excess orbital fat to create a double fold and reduce bulging in the supra-tarsal area [14].

The existing literature shows that several attempts have been made to correct sunken upper eyelids [15-20]. Several surgical methods, including septal fat repositioning, microfat grafting, dermofat grafting, and fascia-fat grafting, as well as nonsurgical methods using materials such as hyaluronic acid gel, silicone bag-gel, and dermal matrix, have been proposed. However, it is difficult to accurately predict the degree of atrophy when using transplanted fat; therefore, the outcomes of surgery may not be consistent, and unsatisfactory results are possible, such as contour irregularity. In addition, the risk of fat embolism, which can lead to stroke or blindness, cannot be completely ruled out $[13,21]$. To overcome these limitations, several studies utilized dermofat grafting and reported good results $[19,20]$. However, this procedure is associated with donor site morbidity, including scarring [21].

In our modified SBB technique, we preserve the subbrow tissue, which is routinely discarded in conventional SBB, as a flap, and utilize the flap to correct sunken deformity (Fig. 5). This technique is simple and can correct sunken deformities without the requirement for an additional donor site. In addition, the recovery time, along with the amount and duration of swelling, associated with our modified technique are not significantly different than those associated with the traditional SBB procedure.

Our flap is not a new concept, and its reliability for the reconstruction of periorbital defects has been reported in many previous studies $[4,5,22,23]$. Considering the successful clinical results reported thus far, as opposed to excision, we have tried to use the skin and orbicularis muscle in the subbrow area as a soft tissue filler to correct sunken deformities.

The main limitation of this study is its retrospective nature and small sample size. Because of this, our data must be interpreted with appropriate care. Notably, a prospective study of patients' and surgeons' satisfaction comparing our technique with conventional techniques (fat graft, dermofat graft, and filler injections) is warranted to ensure that this study is more meaningful in the clinical context. However, compared with the existing techniques used to correct sunken upper eyelid described above, our modified technique is reliable and safe and the risk of infection is low. Elevation of the flap is quite simple, and vascularity can be preserved if dissection is terminated before the supraorbital foramen. In addition, an additional donor site is unnecessary and the volume of augmentation can be adjusted by folding or resecting the flap. 
In conclusion, the best outcomes can only be obtained when a surgical method is selected on the basis of individual patient characteristics. In older patients with a superior sulcus depression, traditional SBB may result in a more visible deformity. In such cases, a de-epithelized orbicularis oculi musculocutaneous flap can produce more reliable and satisfactory results. For surgeons, this modified SBB technique has the advantages of being safe, simple, and easy to learn. We believe that this modified technique can provide the best results for patients requiring simultaneous correction of dermatochalasis and sunken upper eyelid.

\section{NOTES}

\section{Conflict of interest}

No potential conflict of interest relevant to this article was reported.

\section{Ethical approval}

The study was approved by Institutional Review Board of Bucheon St. Mary's Hospital (IRB No. HC18REDI0094) and performed in accordance with the principles of the Declaration of Helsinki.

\section{Patient consent}

The patients provided written informed consent for the publication and the use of their images.

\section{ORCID}

\section{Seong Yeon Kim}

Ma Rhip Ahn

Young Chul Suh

Young Jin Kim

Jung Ho Lee https://orcid.org/0000-0002-2993-1752

https://orcid.org/0000-0001-6222-8842

https://orcid.org/0000-0002-0320-3933

https://orcid.org/0000-0002-9046-9907

https://orcid.org/0000-0002-3800-5494

\section{REFERENCES}

1. Kim YS, Roh TS, Yoo WM, et al. Infrabrow excision blepharoplasty: applications and outcomes in upper blepharoplasty in Asian women. Plast Reconstr Surg 2008;122:1199-205.

2. Hwang K, Kim DJ, Hwang SH. Thickness of Korean upper eyelid skin at different levels. J Craniofac Surg 2006;17:54-6.

3. Tirone L, Schonauer F, Sposato G, et al. Reconstruction of lower eyelid and periorbital district: an orbicularis oculi myocutaneous flap. J Plast Reconstr Aesthet Surg 2009;62:1384-8.

4. Kim SW, Han HH, Jung SN. Orbicularis oculi myocutaneous island flap for upper eyelid reconstruction. J Craniofac Surg 2012;23:746-8.

5. Porfiris E, Tamparopoulos K, Pitsargiotis E, et al. Full-thickness eyelid reconstruction with a single upper eyelid orbicularis oculi musculocutaneous flap. Ann Plast Surg 2006;57:343-7.

6. Paridaens D, van den Bosch WA. Orbicularis muscle advancement flap combined with free posterior and anterior lamellar grafts: a 1-stage sandwich technique for eyelid reconstruction. Ophthalmology 2008; 115:189-94.

7. Porfiris E, Kalokerinos D, Christopoulos A, et al. Upper eyelid island orbicularis oculi myocutaneous flap for periorbital reconstruction. Ophthalmic Plast Reconstr Surg 2000;16:42-4.

8. Fitzgerald R. Contemporary concepts in brow and eyelid aging. Clin Plast Surg 2013;40:21-42.

9. Yun S, Son D, Yeo H, et al. Changes of eyebrow muscle activity with aging: functional analysis revealed by electromyography. Plast Reconstr Surg 2014;133:455e-463e.

10. Osaki MH, Osaki TH, Osaki T. Infrabrow skin excision associated with upper blepharoplasty to address significant dermatochalasis with lateral hooding in select asian patients. Ophthalmic Plast Reconstr Surg 2017;33:53-6.

11. Branham G, Holds JB. Brow/upper lid anatomy, aging and aesthetic analysis. Facial Plast Surg Clin North Am 2015;23:117-27.

12. Lee D, Law V. Subbrow blepharoplasty for upper eyelid rejuvenation in Asians. Aesthet Surg J 2009;29:284-8.

13. Moon HS, Ahn B, Lee JH, et al. Rejuvenation of the deep superior sulcus in the eyelid. J Cosmet Dermatol 2016;15:458-68.

14. Lee W, Kwon SB, Oh SK, et al. Correction of sunken upper eyelid with orbital fat transposition flap and dermofat graft. J Plast Reconstr Aesthet Surg 2017;70:1768-75.

15. Leyngold IM, Berbos ZJ, McCann JD, et al. Use of hyaluronic acid gel in the treatment of lagophthalmos in sunken superior sulcus syndrome. Ophthalmic Plast Reconstr Surg 2014;30:175-9.

16. Cho WK, Jung SK, Paik JS, et al. Surgical outcomes of deep superior sulcus augmentation using acellular human dermal matrix in anophthalmic or phthisis socket. J Craniofac Surg 2016;27:1120-4.

17. Lee S, Yen MT. Nonsurgical rejuvenation of the eyelids with hyaluronic acid gel injections. Semin Plast Surg 2017;31:17-21.

18. Tamboli D, Mancini R. Hyaluronic acid gel injection for multiple eyelid folds in Asian eyelids: a novel approach. Ophthalmic Plast Reconstr Surg 2016;32:310-2.

19. Lin TM, Lin TY, Huang YH, et al. Fat grafting for recontouring sunken upper eyelids with multiple folds in Asians-novel mechanism for neoformation of double eyelid crease. Ann Plast Surg 2016;76:371-5.

20. Lin TM, Lin TY, Chou CK, et al. Application of microautologous fat transplantation in the correction of sunken upper eyelid. Plast Reconstr Surg Glob Open 2014;2:e259.

21. Jeon MS, Jung GY, Lee DL, et al. Correction of sunken upper eyelids by anchoring the central fat pad to the medial fat pad during upper blepharoplasty. Arch Plast Surg 2015;42:469-74.

22. Goldstein MH. "Orbiting the orbicularis": restoration of muscle-ring continuity with myocutaneous flaps. Plast Reconstr Surg 1983;72:294-301.

23. Borman H, Ozcan G. Superomedially based upper eyelid musculocutaneous flap for closure of medial canthal defects. Eur J Plast Surg 2001; 24:239-42. 